\title{
DENDRITIC CELL DIFFERENTIATION BLOCKED BY PRIMARY EFFUSION LYMPHOMA-RELEASED FACTORS IS PARTIALLY RESTORED BY INHIBITION OF P38 MAPK
}

\author{
M. CIRONE, L. DI RENZO, P. TRIVEDI, G. LUCANIA, G. BORGIA, L. FRATI \\ and A. FAGGIONI
}

Istituto Pasteur-Fondazione Cenci Bolognetti, Department of Experimental Medicine University of Rome "Sapienza", Rome, Italy

Received June 18, 2010 - Accepted October 28, 2010

To better understand the molecular mechanisms underlying the dendritic cell (DC) defects in cancer, we analyzed which signaling pathway is implicated in the abnormal monocyte differentiation into DC determined by the presence of Primary effusion lymphoma (PEL) released factors. Our results indicate that the DC, obtained in this condition, together with phenotypic abnormalities and reduced allostimulatory function, showed hyperphosphorylation of signal transducer and activator of transcription 3 (STAT3) and p38 mitogen-activated protein kinase (MAPK) molecules, in comparison to the DC differentiated in the absence of PEL-released factors. The inhibition of p38 MAPK but not of STAT3 phosphorylation, with specific inhibitors, was able to revert the effect of the PEL-released factors on the DC phenotype. This study suggests that p38 MAPK signaling pathway is an important contributor to the abnormal differentiation of DC in PEL.

Primary Effusion Lymphoma (PEL) is an HHV8-associated lymphoma that usually presents as lymphomatous effusion in body cavities. It has an extremely aggressive clinical course with a median survival of only 3 months (1-2). PELs are able to release several inflammatory cytokines such as IL10, IL-6 and VEGF. They act in an autocrine fashion and the PEL growth seems to be dependent on their production (3). These cytokines also determine phosphorylation and subsequent activation of signal trasducer and activator of transcription-3 (STAT3) in PEL (4). STAT3 activation plays a critical role in promoting PEL survival, since it has been reported that its signaling inhibition leads to apoptosis (5). STAT3 is constitutively activated also in other hematological and epithelial cancers (6). In addition,
Wang et al. have shown that STAT3 activation in tumor cells has an essential role in the negative regulation of tumor immunity and in particular on functional activity of DC (7). Tumor cell-released factors inhibit DC maturation by activating STAT3 also in these cells, in a paracrine fashion. DC are the most potent APC (8) and it is known that the function of these cells is impaired in cancer patients (9). Nefedova et al. showed that hyperactivation of STAT3 is responsible for the abnormal in vitro DC differentiation observed in the presence of tumor-derived factors (10) and the inhibition of Jack2/STAT3 signaling is able to restore DC differentiation and function (11). More recently, in a murine model, it was reported that myelomaderived factors impaired the differentiation of bone

Key words:

Mailing address: Mara Cirone, M.D.

Dipartimento di Medicina Sperimentale,

University of Rome "Sapienza",

0394-6320 (2010)

Viale Regina Elena 324,

Copyright $(\odot$ by BIOLIFE, s.a.s.

00161 Roma, Italy

Tel: ++39 064463542 Fax: ++39064468450

e-mail: mara.cirone@uniroma1.it 
marrow (BM)-derived DC and this has been shown by culturing the murine $\mathrm{BM}$ cells with myeloma cells, both in a transwell system and by direct contact, or by adding to the BM cells the tumor culture conditioning medium alone. The myelomaderived factors activate p38 mitogen-activated protein kinase (MAPK) in these BM-derived DC. Its inhibition by the use of specific compounds was able to restore the generation of functional DC and these results indicate that $\mathrm{p} 38$ pathway is a major contributor to the DC differentiation abnormalities (12). Similar results were obtained with human monocyte-derived DC from patients affected by multiple myeloma (13). We previously reported that soluble factors released by PEL inhibit DC differentiation from monocyte precursors and impair their function (14). In this study we investigated which signal transduction pathway is involved in PEL-induced suppression of DC differentiation and whether any pharmacological treatment could revert the effect of the PEL supernatant. We found that, in the presence of PEL-released factors, the DC display hyperphosphorylation of STAT3 and p38 MAPK molecules. With the use of their specific inhibitors, we investigated whether the phenotype and the function of DC could be restored. The results obtained indicate that p38 inhibitor SB203580 was able to revert the inhibition of DC differentiation determined by PEL supernatant but, since only the phenotype but not the function was restored, we conclude that other molecules play a role in the DC abnormalities in PEL. It has been reported that DC function depends on NF-kappa B activity (15) and since STAT3 and p38 MAPK have a negative effect on NF-kappaB $(8,16)$ we investigated the activation state of this molecule in DC differentiated in the presence and in the absence of PEL supernatant with or without STAT3 and p38 MAPK specific inhibitors.

\section{MATERIALS AND METHODS}

\section{Cells and antibodies}

The PEL cell lines BC-3, JSC-1 and BCP1 were seeded at $1 \times 10^{5}$ and cultured in RPMI $164010 \%$ Fetal Calf Serum (FCS) (Euroclone). After 4 days of culture, the cells were centrifuged at $1500 \mathrm{RPM}$ for $10 \mathrm{~min}$ and the supernatants were filtered through a $0.2 \mu \mathrm{m}$ filter. Monoclonal antibodies used for FACS analysis and immunofluorescence were anti-CD14 and anti-CD1a (Becton-Dickinson) and anti-NF-kappaB p65 (Santa Cruz). Monoclonal antibodies used for western blotting were anti-phosho-p38 MAPK, anti-p38 MAPK, antiphospho-ERK and anti-ERK (Cell Signaling), anti- $\beta$-actin (Sigma) anti-phospho-STAT3 Ty705 and anti-STAT3 total protein (BD Bioscience).

\section{Generation of monocyte-derived DC}

To generate monocyte-derived DC, human peripheral blood mononuclear cells were isolated by FycollPaque gradient centrifugation (Pharmacia, Uppsala, Sweden) from buffy coats. CD14+ monocytes were positively selected using anti-CD14 MAb-conjugated magnetic microbeads (Miltenyi Biotec, Auburn, Calif.). Purified monocytes were cultured at a density of $1 \times 10^{6}$ cells $/ 3 \mathrm{ml}$ in 12-well plates for 6 days in RPMI 1640 (Euroclone) containing 10\% FCS, $2 \mathrm{mM}$ L-glutamine, $100 \mathrm{U} / \mathrm{ml}$ penicillin $\mathrm{G}, 100 \mathrm{mg} / \mathrm{ml}$ streptomycin, $50 \mathrm{mM}$ 2-mercaptoethanol (Sigma), and recombinant human granulocyte-macrophage colony stimulating factor (GM-CSF) plus interleukin 4 (IL-4) $(50 \mathrm{ng} / \mathrm{ml}$ and 20 $\mathrm{ng} / \mathrm{ml}$ respectively, Miltenyi Biotec, Auburn, Calif.). PEL supernatants were filtered through $0.2 \mu \mathrm{m}$, analyzed by PCR to exclude the presence of HHV-8 and added to the monocyte culture on day 0 at concentration of $20 \%$. Cytokines were replenished every other day by adding $20 \%$ fresh medium to each well.

Blocking STAT3 phosphorylation using Janus-Activated Kinase 2 inhibitors AG490 and JSI-124

To study the effect of blocking STAT3 activation during the DC differentiation in the presence of PEL supernatants, $\mathrm{CD} 14^{+}$monocytes were pretreated with AG490 at $10 \mu \mathrm{mol} / \mathrm{L}$ for $2 \mathrm{~h}$, the cells were then washed with PBS and resuspended in the regular DC differentiation cocktail plus PEL supernatants (i.e., complete RPMI 1640 with GM-CSF and IL-4) for 6 days. JSI-124 (Sigma-Aldrich) inhibitor was used at $0.5 \mu \mathrm{M}$, added to the differentiation cocktail on day 4 and kept for 24 hours. Cell number was determined by viable cell count. AG490 had no effect whereas JSI-124 reduced the number of cellS recovered.

\section{Blocking p38 and ERK phosphorylation}

The role of $\mathrm{p} 38$ and ERK signaling pathways was investigated by blocking p38 MAPK with SB203580 and with PD98059 at $10 \mu \mathrm{M}$ (Sigma-Aldrich). Their concentration was chosen based on previous studies (16) where the inhibitors were titrated for their capacity to inhibit the activity of the kinases. Both inhibitors were incubated for $30 \mathrm{~min}$ at $37^{\circ} \mathrm{C}$ before culturing monocytes with differentiation cocktail plus PEL supernatant for 6 
days. SB 203580 did not have any effect on the cell yield whereas PD98059 reduced the cell number.

\section{Immunoblotting}

After 6 days of culture, DC were lysed with $100 \mu \mathrm{L}$ lysis buffer (Cell Signaling Technology). The lysates (100 $\mu \mathrm{g})$ were then resolved by SDS-PAGE and transferred to nitrocellulose membranes (Bio-Rad). The levels of the phosphorylated STAT3, p38MAPK, ERK were detected using phospho-specific antibodies. Antibodies directed against the total proteins and $\beta$-actin were also used and the reactions were visualized by using a goat antimouse horseradish peroxidase conjugate antibody (Cell Signaling Technology) and enhanced chemiluminescence (Amersham).

\section{Flow cytometric analysis}

For immunophenotyping, cells were washed in PBS and incubated for $30 \mathrm{~min}$ at $4^{\circ} \mathrm{C}$ with the following monoclonal antibodies: CD14 and CD1a (Becton and Dickinson). For the unconjugated antibodies the binding was revealed by goat FITC-conjugated anti-mouse Ig (Cappel). The samples were analyzed using a FACScan cytometer (Becton and Dickinson).

\section{Mixed lymphocyte reaction}

MLR were performed on immature DC obtained after 6 days of culture or on mature DC obtained by adding LPS from Salmonella abortus equi $(1 \mu \mathrm{g} / \mathrm{ml})$ for an additional 48 hours. The assay was carried out in 96-well flat-bottom microtiter plates by adding different numbers of irradiated $(3000 \mathrm{rad})$ DC to $2 \times 10^{5}$ allogeneic PBL. At day 6 of culture at $37^{\circ} \mathrm{C}$, cell proliferation was assessed by uptake of ${ }^{3} \mathrm{H}$ thymidine $(1 \mu \mathrm{Ci} / \mathrm{well})$ added for the last 16 hours.

\section{Immunofluorescence}

After 6 days of culture, DC were washed with PBS and fixed with absolute methanol for $5 \mathrm{~min}$ at $-20^{\circ} \mathrm{C}$. Cells were then incubated for $1 \mathrm{~h}$ with rabbit polyclonal antibody to NFkB p65 (Santa Cruz), rinsed 3 times with PBS and then incubated with FITC-conjugated anti-rabbit $\operatorname{IgG}$ (Sigma) for $1 \mathrm{~h}$. Cells were then rinsed 3 times with PBS and mounted with Prolong anti-fast reagent and the fluorescence was analysed by an Olympus BX52 fluorescence microscope. The images were acquired and elaborated with IAS 2000 software.

\section{RESULTS}

PEL-released factors hyperphosphorylate STAT3 and $p 38 M A P K$ in monocyte derived DC

The cytokines IL-6, IL-10 and VEGF released by PEL cell lines and detected in PEL supernatants are known to converge in hyperphosphorylation of STAT3, in an autocrine fashion. To verify whether they also hyperphosphorylate STAT3 in monocytederived DC in a paracrine fashion, we performed a western blot analysis using a specific antibody against p-STAT3. As shown in Fig. 1, the DC obtained in the presence of PEL (BC3) supernatant showed an increase of STAT3 tyrosine-phosphorylation at Tyr705 after 6 days of culture when compared to the DC differentiated in the absence of PEL supernatant. Similar results were obtained with three different donors and two additional PEL supernatants (JSC1 and BCP1, data not shown). We then blocked STAT3 hyperphosphorylation in monocyte-differentiating DC by using a JAK2/STAT3 inhibitor AG 490 at 10 $\mathrm{mM}$ for 2 hours before starting the culture with GMCSF and IL-4 plus the PEL supernatant. On day 6 of culture we performed a western blot analysis also on DC obtained in the presence of AG490 and the results obtained indicate that this inhibitor was able to reduce STAT3 phosphorylation induced by PEL supernatant (Fig. 1). Similar results were obtained with JSI-124, which was added to the CD14 culture on day 4 of culture at $0.5 \mathrm{mM}$ and kept for the last 48 hours (data not shown). Other abnormal signaling pathways are reported to be associated with tumor inhibition of DC differentiation such as p38 MAPK pathway that seems to be the most important pathway in the DC dysfunction in multiple myeloma (12-13). We investigated the protein level of phosphorylated p38 MAPK and ERK in the DC obtained in the presence or in the absence of PEL supernatants. The results shown in Fig. 2 indicate that DC obtained in the presence of PEL supernatant, display an increase of p38 MAPK phosphorylation while no modification of ERK phopsphorylation was observed. The effect of p38 MAPK specific inhibitor SB203580 on p38 phosphorylation is also shown. The lack of quantitative difference in pp38 levels in SB203580treated DC is most probably due to the fact that this inhibitor does not block the phosphorylation but inhibits the kinase activity (13).

P38 MAPK but not Jak2/STAT3 specific inhibitor partially reverts the effect of PEL supernatant on DC differentiation

To determine the causal relationship between 


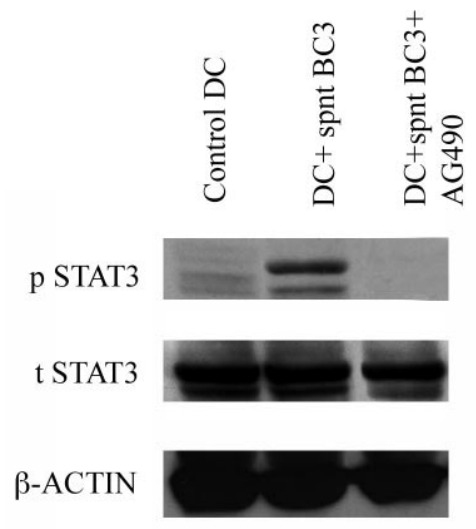

Fig. 1. STAT3 activation in PEL-mediated blocking of $D C$ differentiation. Level of STAT3 phosphorylation ( $p$ STAT3) in the DC differentiated in the absence, in the presence of PEL supernatant or in the presence of PEL supernatant plus Jak2/STAT3 inhibitor AG490. Level of total STAT3 (t-STAT3) and $\beta$-actin is also shown. Data for one of three representative donors is shown.

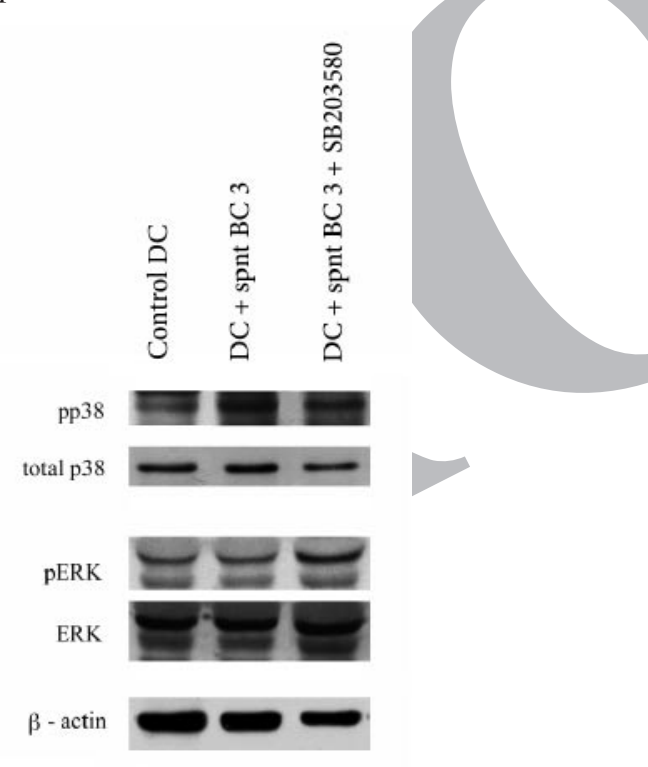

Fig. 2. $p 38 M A P K$ activation in PEL-mediated blocking of DC differentiation. Level of p38 MAPK and ERK phosphorylation in the DC differentiated in the absence or in the presence of PEL supernatant or in the presence of PEL supernatant plus p38 MAPK inhibitor SB203580. Level of total $p 38$ and ERK molecules and $\beta$-actin is also shown. Data for one of three representative donors is shown.

the abnormal signaling and the impairment of DC differentiation observed in the presence of PEL released factors, we pretreated $\mathrm{CD} 14^{+}$monocytes with Jak2/STAT3 inhibitor AG490 or p38 MAPK inhibitor SB203580 before exposure to the PEL supernatant and to the differentiation cocktail. To answer the question whether the inhibition of DC differentiation observed in the presence of PEL supernatant could depend on STAT3 and/or p38 MAPK hyperphosphorylation, we performed a FACS analysis on DC obtained in the presence of PEL supernatant with or without inhibitors. We analyzed the surface expression of molecules modified during the monocyte differentiation into DC in the presence of PEL-released factors and in particular the expression of CD1a, which was strongly reduced and CD14, which was not lost in the DC differentiated in the presence of PEL supernatant (Fig. 3C). The results obtained with the use of kinase inhibitors indicate that, while the inhibition of p38 MAPK with SB203580 partially reverted the effect of PEL supernatant on these differentiation markers (Fig. 3D), AG490 did not modify the expression of CD14 and CD1a (Fig. $3 \mathrm{E})$. The mean percentage positive cells plus standard deviation (SD) of four experiments is the following: control DC-81.6 \pm 15 , spnt-treated DC- $19.5 \pm 11$, spntDC plus SB203580-56 \pm 20 and spnt-DC plus AG$49027.2 \pm 6$. No significant modification on the CD83 and 86 costimulatory molecules was observed in the presence or in the absence of PEL supernatant or with the kinase inhibitors (data not shown). To investigate whether inhibition of STAT3 or p38 MAPK phosphorylation could have also an effect in restoring DC function, we performed an MLR assay on the DC treated with PEL supernatant in the presence or in the absence of AG490 and SB204580. As shown in Fig. 4a, the DC obtained in the presence of both inhibitors, alone or in combination, did not recover the allostimulatory capacity reduced by PEL. Nor was the allostimulatory function of DC restored after their complete maturation with LPS (Fig. 4b).

\section{Effect of PEL-released fctors on NF-kappaB activation on monocyte derived $D C$}

Since NF-kappaB activation is essential for the allostimulatory activity of the DC, we evaluated its status in DC. The active NF-kappaB translocates into the nucleus from the cytoplasm where it is present in an inactive form. In these experiments we performed immunofluorescence detection of the Rel A subunit of NF-kappaB since it has been reported that p50 and RelA may be the most important NF- 

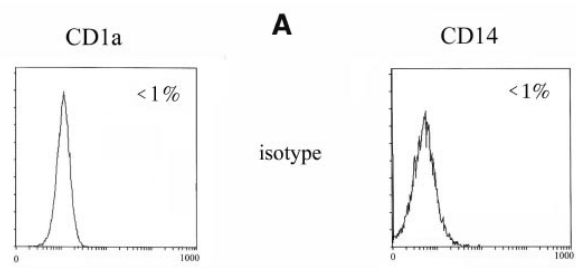

B

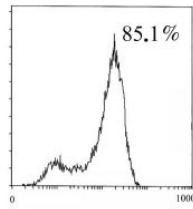

control DC

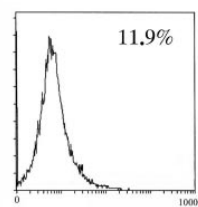

C

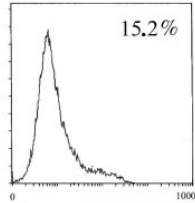

$\mathrm{DC}+$ spnt $\mathrm{BC} 3$

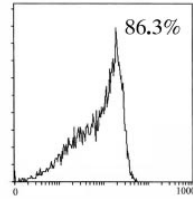

D

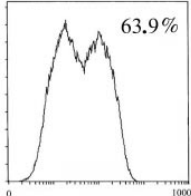

$\mathrm{DC}+$ spnt $\mathrm{BC} 3$ + SB203580

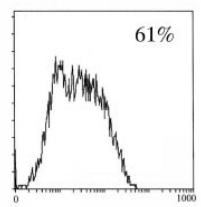

E

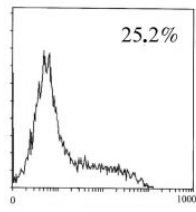

$\mathrm{DC}+$ spnt $\mathrm{BC} 3$ $+\mathrm{AG} 490$

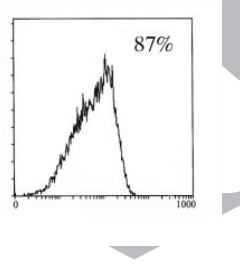

Fig. 3. Effect of PEL released factors in the presence or in the absence of $A G 490$ and SB203580 on DC differentiation markers. CDIa and CD14 immunofluorescence were detected by flow cytometry. A: isotype control, B: DC obtained with a cocktail of IL-4 and GM-CSF, C: DC obtained in the presence of $20 \%$ BC3 supernatant, D: As in $C+S B 203580, E:$ As in $C+A G 490$. One representative experiment out of four is shown. The numbers represent the percentage of positive cells.

kappaB subunits in developing DC (17).

As shown in Fig. 5a, NF-kappaB is activated and translocates to the nuclei in $20 \%$ of control DC while in DC obtained in the presence of PELreleased factors it was retained in the cytoplasm in approximately all of the cells (Fig. 5b). We further investigated whether the use of AG490 or SB203580 could restore NF-kappaB traslocation and activation inhibited by PEL supernatant. In Fig. $5 \mathrm{c}$ and d, we show that the use of AG490 or SB203580 was not sufficient to reactivate NF-kappaB and probably this can be one of the reasons why MLR function was not resored in these DC.

\section{DISCUSSION}

It is well-documented that differentiation and function of DC in cancer patients are impaired due to the tumor cells or tumor-derived cytokines and other released factors (18-19). The defective differentiation of tumor patient-derived DC could be due also to defects in the progenitor cells from patients. Studies on PEL patient ex vivo-generated DC are lacking. We have recently shown that supernatants of PEL cell lines are able to inhibit the in vitro differentiation of DC from $\mathrm{CD} 14^{+}$monocytes. In our study, the abnormal DC differentiation is observed utilizing, as precursors, monocytes derived from healthy donors and the inhibition of DC differentiation is completely due to the PELreleased factors and in particular to IL-6, IL-10 and VEGF (14). Their role has been highlighted by the use of neutralizing antibodies. These cytokines converge in the hyperphosphorylation of STAT3 and the consequent aberrant activation of STAT3 plays a pivotal role in the negative regulation of antitumor immunity $(10,18)$. Other pathways, such as p38 MAPK, can be activated by tumor-released factors and interfere with DC differentiation, as reported for multiple myeloma (12). In the present work, we show that PEL-released factors hyperphosphorylate STAT3 and p38 MAPK molecules in monocytederived DC. To investigate which signaling pathway plays a major role in the defect of DC differentiation and function, specific inhibitors for kinases were used. SB203580 (p38 inhibitor), but not AG490 (STAT3 inhibitor), was able to restore the monocyte differentiation into DC, as indicated by the CD1a expression. However, neither AG490 nor SB203580 were able to restore the DC function and in particular the MLR capacity. These results suggest that additional pathways are involved in the upregulation of CD1a molecule and the function of DC and that at least the MLR capacity probably correlate more with CD86 and CD83 expression, which were indeed not modified by p38 and STAT3 inhibitors (data not 

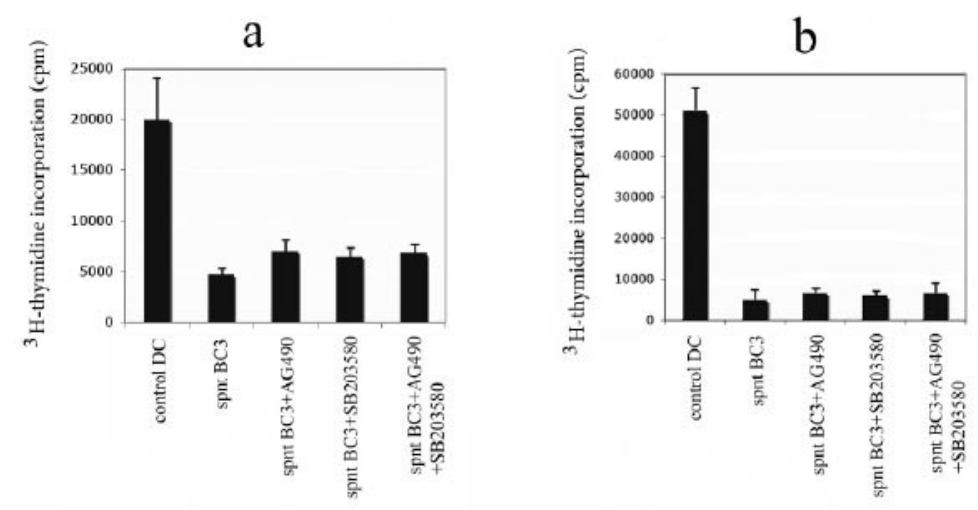

Fig. 4. Mixed Lymphocyte Reaction (MLR) of immature (a) and mature DC (b) differentiated in the absence or in the presence of PEL released factors with or without AG490 or SB203580. Each bar represents the mean plus the standard deviation of $3 H$-thymidine incorporation of three different experiments. Ratio DC:T=1:10
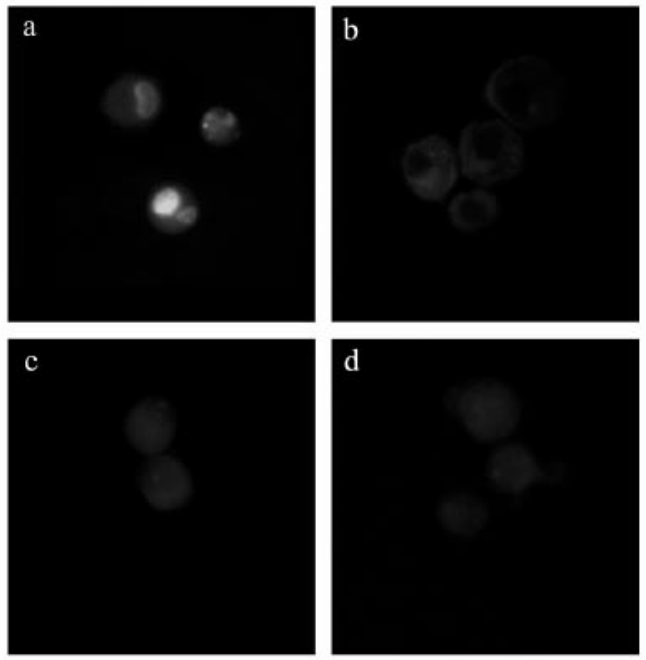

Fig. 5. NF-kappaB activity in DC differentiated in the absence (a) or in the presence (b) of PEL released factors with AG490 (c) and SB203580 (d). Immunofluorescence detection of Rel A subunit of NF-kappaB. One representative experiment out of two is shown.

shown). In addition, the presence of the above inhibitors did not reactivate NF-kappaB, which resulted inhibited and remained in the cytoplasm in the DC differentiated in the presence of PELreleased factors. It was previously reported, with different approaches, that allostimulatory function of DC is dependent on NF-kappaB activation (20). We were not able to see activation of NF-kappaB with either of the two inhibitors and this suggests that tumors factors other than 38 MAPK and STAT3 are involved in the inhibition of NF-kappaB. STAT3 is crucial for tumor-cell proliferation and survival for many tumors, including PEL (5), and its constitutive activation can be propagated from tumor cells to the cells of the immune system with inhibitory effect on its function. Indeed, it has been reported that, besides acting as a chemotherapeutic drug, STAT3 inhibitors can improve the DC activity against some types of tumors (7). However, a previous paper on DC defect in multiple myeloma showed that, in spite of the presence of hyperphosphorylated STAT3, the activation of p38 MAPK signaling pathways, together with inhibition of MEK/ERK, is the major contributor to the abnormalities of these monocytederived DC (12). Our results that $\mathrm{p} 38$ MAPK pathway is more important than STAT3 for the inhibition of DC differentiation in PEL are consistent with similar observation in myeloma and are also in agreement with previous studies showing that p38 MAPK activation inhibits DC differentiation (16). On the contrary, during the maturation, its activation has a positive role (21-22). Moreover, we found that the PEL-released factors inhibited NF-kappaB molecule, which is normally activated and translocated to the nucleus in monocyte-derived DC (16). Removing the inhibitory effect of STAT3 and p38 by using AG490 and SB203580 was not sufficient to restore NF-kappaB activation. Progress in signaling pathways involved in DC maturation has been made 
over the past several years, while the molecular events involved in the differentiation and generation of immature DC are still poorly characterized. This study provides important insight into the molecular mechanisms regulating the DC differentiation and in particular its inhibition in the presence of soluble factors released by PEL. Further studies are needed to clarify the molecular pathways underlying the molecular defects of DC found in cancer patients (23) since DC-based immunotherapy holds great promise for treating human malignancies.

\section{ACKNOWLEDGEMENTS}

This work was partially supported by grants from MIUR, Associazione Italiana per la ricerca sul Cancro (AIRC), progetto strategico ISS 9ACF/1 of Ministero della salute, Fondazione Italiana per la sclerosi multipla (2007/R/17), Pasteur CenciBolognetti foundation. We thank Mr. Sandro Valia for help with photographic work.

1. Boshoff C, Weiss R. AIDS-related malignancies. Nat REFERENCES

Rev Cancer 2002; 2:373-82.

2. Nador RG, Cesarman E, Chadburn A, Dawson DB, Sald J, Knowles DM. Primary effusion lymphoma: a distinct clinicopathologic entity associated with the Kaposi's sarcoma-associated herpes virus. Blood 1996; 88:645-56.

3. Ensoli B, Stürzl M, Monini P. Cytokine-mediated growth promotion of Kaposi's sarcoma and primary effusion lymphoma. Seminars in Cancer Biology 2000; 10:367-81.

4. O'Shea JJ, Gadina M, Schreiber RD. Cytokine signaling in new surprises in the Jak/Stat pathway. Cell 2002; 109:S121-31.

5. Aoki Y, Feldman GM, Tosato G. Inhibition of STAT3 signaling induces apoptosis and decreases survivin expression in primary effusion lymohoma. Blood 2003; 101:1535-42.

6. Brombrg J. Stat proteins and oncogenesis. J Clin Invest 2000; 109:1139-42.

7. Wang T, Niu G, Kortylewski M, et al. Regulating of the innate and adaptive immune responses by Stat-3 signaling in tumor cells. Nat Med 2004; 10:48-54.
8. Gottfried E, Kreutz M, Mackensen A. Tumorinduced modulation of dendritic cell function. Cytokine Growth Factor Rev 2008; 19:65-77.

9. Banchereau J, Bruire F, Caux C, Davoust J, Lebecque S, Liu Y, Pulendran B, Paluda K. Immunobiology of dendritic cells. Annu Rev Immunol 2000; 18:767811.

10. Nefedova Y, Huang M, Kusmartsev S, Battacharya R, Cheng P, Salup R, Jove R, Gabrilovich D. Hyperactivation of STAT3 is involved in abnormal differentiation of dendritic cells in cancer. J Immunol 2004; 172:464-74.

11. Nefedova Y, Cheng P, Gilkes D, Blaskovich M, Beg AA, Sebti SM, Gabrilovich D. Activation of dendritic cells via inhibition of Jak2/STAT3 signaling. J Immunol 2005; 175:4338-64.

12. Wang S, Yang J, Qian J, Wezeman M, Kwak LW, Yi Q. Tumor evasion of the immune system: inhibiting p38 MAPK signaling restores the function of dendritic cells in multiple myeloma. Blood 2006; 107:2432-9.

13. Wang S, Hong S, Yang J, Qian J, Zhang X, Shpall E, Kwak LW, Yi Q. Optimizing immunotherapy in multiple myeloma: Restoring the function of patients' monocyte-derived dendritic cells by inhibiting p38 or activating MEK/ERK MAPK and neutralizing interleukin-6 in progenitor cells. Blood 2006; 108:4071-7.

14. Cirone M, Lucania G, Aleandri S, Borgia G, Trivedi P, Frati L, Faggioni A. Suppression of dendritic cell differentiation through cytokines released by Primary Effusion Lymphoma. Immunol Lett 2008; 120:37-41.

15. Yoshimura S, Bondeson J, Brennan FM, Foxwell BM, Feldmann M. Role of NFkappaB in antigen presentation and development of regulatory $\mathrm{T}$ cells elucidated by treatment of dendritic cells with the proteasome inhibitor PSI. Eur J Immunol 2001; 31: 1883-93.

16 Xie J, Qian J, Yang J, Wang S, Freeman III ME, Yi Q. Critical roles of Raf/MEK/ERK and PI3K/AKT signaling and inactivation of $\mathrm{p} 38$ MAP kinase in the differentiation and survival of monocyte-derived immature dendritic cells. Exp Hemathology 2005; 33:564-72.

17. Yongwon Choi, Beg AA. Dendritic Cell Development 
and Survival Require Distinct NF-kappaB Subunits. Immunity 2002; 16:257-70.

18. Bharadwaj U, Li M, Zhang R, Chen C, Yao Q. Evated interleukin-6 and G-CSF in human pancreatic cancer cell conditioned medium suppress dendritic cell differentiation and activation. Cancer Res 2007; 67: 5479-88.

19. Orsini E, Guarini A, Chiaretti S, Mauro FR, Foa R. The circulating dendritic cell compartment in patients with chronic lymphocytic leukemia is defective and unable to stimulate an effective T-cell response. Cancer Res 2003; 63:4497-506.

20. Yoshimura S, Bondeson J, Foxwell BMJ, Brennam FM, Feldmann M. Effective antigen presentation by dendritic cells is NF-kappaB dependent: coordinate regulation of MHC, co-stimulatory molecules and cytokines. Int Immunol 2001; 13:675-83.

21. Arrighi JF, Rebsamen M, Rousset F, Kindler V, Hauser C. A critical role for p38 mitogen-activated protein kinase in the maturation of human bloodderived dendritic cells induced by lipopolysaccharide, TNF-alpha, and contact sensitizers. J Immunol 2001, 166:3837-45.

22. Pan K, Wang H, Liu W, et al. The pivotal role of p38 and NF-kB signal pathways in the maturation of human monocyte-derived dendritic cells stimulated by streptococcal agent OK-432. Immunobiol 2009; 217:350-8.

23. Melief CJM. Cancer immunotherapy by dendritic cells. Immunity 2008; 29:372-83. 